\title{
Techniques for Percutaneous Transesophageal Gastro-tubing in the Management of Gastric Leak or Dysphasia After Sleeve Gastrectomy
}

\author{
Takashi Oshiro ${ }^{1}$ (1) - Taiki Nabekura ${ }^{1} \cdot$ Tomoaki Kitahara $^{1}$ - Ayako Takenouchi ${ }^{1} \cdot$ Yuki Moriyama $^{1} \cdot$ Natsumi Kitahara $^{1}$. \\ Makoto Nagashima ${ }^{1} \cdot$ Shinichi Okazumi ${ }^{1}$
}

Received: 14 December 2021 / Revised: 12 January 2022 / Accepted: 14 January 2022 / Published online: 20 January 2022

(c) The Author(s) 2022

Keywords Percutaneous transesophageal gastro-tubing (PTEG) · Double PTEG · Sleeve gastrectomy · Obesity

\section{Introduction}

Sleeve gastrectomy (SG) has become the most widely performed bariatric and metabolic surgery worldwide [1] owing to its technical simplicity and favorable weight loss effects. However, a tube-shaped stomach after sleeve gastrectomy could be a negative factor that retards healing of leakage [2] and hinders the creation of percutaneous endoscopic gastrostomy.

Although the nasogastric tube (NGT) is a useful tool for managing sleeve leakage and feeding enteral nutrition, it is not appropriate for long-term placement because of poor patient tolerability and potential complications [3].

Percutaneous transesophageal gastro-tubing (PTEG) is a viable alternative to nasogastric decompression or enteral

Key Points

- PTEG is an alternative access route into the gastrointestinal tract using the transesophageal approach.

- An advantage of double PTEG is long-term use for enteral feeding and drainage, with reduced patient burden.

- PTEG or double PTEG has potential application in the management of complications in obese patients after sleeve gastrectomy.

Takashi Oshiro

oshiro@sakura.med.toho-u.ac.jp

Taiki Nabekura

taiki.nabekura@med.toho-u.ac.jp

Tomoaki Kitahara

kita@sakura.med.toho-u.ac.jp

Ayako Takenouchi

ayako.takenouchi@med.toho-u.ac.jp

Yuki Moriyama

yuuki.01.moriyama@med.toho-u.ac.jp access in patients who require long-term tube placement [4]. Furthermore, one additional indwelling tube through the same opening of the primary PTEG (double PTEG) simultaneously enables enteral nutrition access and gastric drainage separately [5].

\section{Materials and Methods}

We experienced 4 ( 1 male, 3 female) cases of PTEG in patients after SG (3 for early sleeve leakage and 1 for dysphagia due to amyotrophic lateral sclerosis (ALS): case 3). PTEG for sleeve leakage was performed following the failure of endoscopic treatments including clipping and stenting. One patient underwent double PTEG due to refractory sleeve leakage (case 4). The first and third cases were performed under local anesthesia and intravenous sedation, while the two other cases were subjected to general anesthesia for postural stability.

The PTEG kit was supplied by SB-Kawasumi Laboratories, Inc. (Tokyo, Japan). The procedure was performed as previously described by Oishi et al. [6]. Briefly, the procedure was performed as follows. A tube with rupture

\author{
Natsumi Kitahara \\ natsumi.kitahara@med.toho-u.ac.jp \\ Makoto Nagashima \\ nagashima@sakura.med.toho-u.ac.jp \\ Shinichi Okazumi \\ sokazumiyrsh@sakura.med.toho-u.ac.jp \\ 1 Department of Surgery, Toho University Sakura Medical \\ Center, 564-1 Shimoshizu, Sakura, Chiba 285-8741, Japan
}


free balloon (RFB) was inserted into the upper esophagus through a nostril. The RFB was prepared using chloroethylene to prevent its rupture by needle puncture and to increase its ultrasonographic visibility. Inflated RFB with mixed contrast medium and saline was punctured percutaneously using an introducer needle through the left side of the neck under ultrasonography and fluoroscopy. A matching guidewire was passed through the needle into the RFB, and then threaded into the esophageal lumen following RFB tube removal. After dilating the path around the guidewire using a strong and flexible dilator, the indwelling tube was advanced over the guidewire. An additional intraluminal tube was inserted through the same opening of the primary PTEG using a second guidewire and a dilator with a peel-away sheath. The double PTEG technique is shown in Video S1 (case 4).

\section{Results}

At the time of PTEG, the body mass index (BMI) of each of the four patients was $35 \mathrm{~kg} / \mathrm{m}^{2}, 40 \mathrm{~kg} / \mathrm{m}^{2}, 30 \mathrm{~kg} / \mathrm{m}^{2}$, and $43 \mathrm{~kg} / \mathrm{m}^{2}$, respectively, and BMI loss from pre-SG was $9 \mathrm{~kg} /$ $\mathrm{m}^{2}, 19 \mathrm{~kg} / \mathrm{m}^{2}, 14 \mathrm{~kg} / \mathrm{m}^{2}$, and $8 \mathrm{~kg} / \mathrm{m}^{2}$, respectively. PTEG was successful in all patients without any adverse events and enabled tube feeding the following day. The tube was removed without any complications in the cases of sleeve leakage. Two of them were healed by the timing of the tube removal on post-PTEG day 96 for case 1 and 43 for case 2 . Although intractable sleeve leakage (case 4 ) required revision surgery for laparoscopic total gastrectomy 3 months after double PTEG, long-term tube feeding did not necessitate parenteral nutrition, and another intraluminal drainage tube with intermittent suction could contribute to infection control. The orifice of the tubing at the left neck was completely closed within a few days after tube removal. Patients with ALS received enteral nutrition support using PTEG for over a year without any complications.

\section{Conclusion}

PTEG (or double PTEG) may be considered as a potential therapeutic option to overcome the difficulties resulting from a sleeve-like stomach after SG. PTEG could be an alternative to NGT for long-term placement and minimize the burden on patients requiring long-term tube placement. Appropriate patient selection for balanced benefit from PTEG is challenging in obese patients needing bariatric and metabolic surgery.

Supplementary Information The online version contains supplementary material available at https://doi.org/10.1007/s11695-022-05909-0.

\section{Declarations}

Ethics Approval All procedures performed in studies involving human participants were in accordance with the ethical standards of the institutional and/or national research committee and with the 1964 Helsinki Declaration and its later amendments or comparable ethical standards. This article does not contain any studies on animals performed by any of the authors. This was a retrospective study, and for this type of study, formal consent was not required.

Consent to Participate Informed consent was obtained from all individual participants included in this study. No identifying information is available in this article.

Conflict of Interest The authors declare no competing interests.

Open Access This article is licensed under a Creative Commons Attribution 4.0 International License, which permits use, sharing, adaptation, distribution and reproduction in any medium or format, as long as you give appropriate credit to the original author(s) and the source, provide a link to the Creative Commons licence, and indicate if changes were made. The images or other third party material in this article are included in the article's Creative Commons licence, unless indicated otherwise in a credit line to the material. If material is not included in the article's Creative Commons licence and your intended use is not permitted by statutory regulation or exceeds the permitted use, you will need to obtain permission directly from the copyright holder. To view a copy of this licence, visit http://creativecommons.org/licenses/by/4.0/.

\section{References}

1. 5th-IFSO Global Registry Report 2019 [Internet]. [cited 2021 Nov. 18]. Available from: https://www.ifso.com/pdf/5th-ifsoglobal-registry-report-september-2019.pdf

2. Praveenraj P, Gomes RM, Kumar S, et al. Management of gastric leaks after laparoscopic sleeve gastrectomy for morbid obesity: a tertiary care experience and design of a management algorithm. J Minim Access Surg. 2016;12(4):342-9.

3. Oshiro T, Saiki A, Suzuki J, et al. Percutaneous transesophageal gastro-tubing for management of gastric leakage after sleeve gastrectomy. Obes Surg. 2014;24(9):1576-80.

4. Udomsawaengsup S, Brethauer S, Kroh M, et al. Percutaneous transesophageal gastrostomy (PTEG): a safe and effective technique for gastrointestinal decompression in malignant obstruction and massive ascites. Surg Endosc. 2008;22(10):2314-8.

5. Tamamori Y, Sakurai K, Kubo N, et al. Percutaneous transesophageal gastro-tubing for the management of anastomotic leakage after upper GI surgery: a report of two clinical cases. Surg Case Rep. 2020;6(1):214-20.

6. Oishi H, Shindo H, Shirotani N, et al. A nonsurgical technique to create an esophagostomy for difficult cases of percutaneous endoscopic gastrostomy. Surg Endosc. 2003;17(8):1224-7.

Publisher's Note Springer Nature remains neutral with regard to jurisdictional claims in published maps and institutional affiliations. 